\title{
II problema della trasformazione artificiale delle condizioni meteorologiche $(*)$
}

\author{
A. SFIRRA (**) \\ Ricevuto il 19 Fehbraio 1963
}

Rusicusto. -..- Sono esposti brevemente i principali piani di esperienza di laboratorio e campali realizzati dal 1947 al 1960 relativamente alla modificazione artificiale delle condizioni meteorologiche in generale e della induzione artificiale delle precipitazioni atmosferiche.

Successivanente sono descritti i principi su cui sono fondate le tecniche piì note sulle operazioni di induzione provocata delle precipitazioni atmosferiche. Infue sono trattati brevemente gli aspetti del sudletto problema ancora aperti alla ricerea e le varie possibilita pratiche di intervento dell'uomo sulla modificazione artificiale telle condizioni meteorologiche.

Somury. We review in brief the main plans of laboratory and field tests carricel ont in the world from 1947 to 1960. in oreler to obtain artificial modifications of meteorological conditions in general and artificial induction of atmospheric precipitations.

Successively, we relate on the principles on which the most well known techuiques of artificial induction operations of atmospheric precipitations are founded.

At last. we deal briefly with aspects of the aforesaid problem yet open to researeh and the varions pratical possibilities of human intervention on artifieial modifieation of meteorologieal conditions.

\section{I. - INTRODUZIONE.}

E di vecehia data l'ambizioso sogno dell'nomo di soggiogale alla sua volontà le immense forze della natura, che governano le condizioni

(*) Nota presentatal al 20 Congresso Internazionale Ternico serentifico dello spazio. Roma, 19-23 Giugno 1962.

(**) Osservatorio seientifieo sperimentale di Heteonologia Aeronantioa di Caglialri. 
meteorologiche e la loro evoluzione. Tuttavia la impostazione su basi scientifiche di un tale problema, che tanto fervore di ricerehe oggi suscita nel mondo, risale a pooco più di due decenni, rios alle prime esperienze di labonatorio e campali eftettuate da I. Langmuir (') e V. J. Schatefer (-), nel 1946 negli C.S.S. I tentativi fatti, anche in tempi relativamente recenti, come alla fine del secolo seorso ed agli inizi dell'attuale, da J. P. Espy $\left(^{3}\right)$, F. Powers (4). I. (Gathman $\left(^{5}\right)$ negli U.S.A., A. W. Veratat $\left(^{6}\right)$ in Olanda, sebbene possano vantare una certa priorità nell'uso di tecniche arboperate suceessivamente con maggior fortuma, mancarono sempre di una arleguata preparazione scientifica e si ridussero a tentativi spoladlici senza suceesso. Ia soluzione del problema io intimamente legata alla conoscenza delle legggi che regolano fenomeni complessi, non ancoma pienamente comosciuti della fisica delle nubi e delle precipitazioni, degrli scambi energetici nell'atmosfera, della circolazione atmosferica. Ia conoscenza di tali fenomeni deve, necessariamente, essere estesa oltre i limiti della troposfera essendo essi collegati ad altri fenomeni che arvengono in zone sempre più vaste dell'alta atmosfera e dello spazio cosmiceo.

Il vasto campo della " modifieazione artificiale delle condizioni meteorologiche" si sudulivide, a sua volta, in "ampi che, sebbene di più limitata portata, sono tuttavia di enorme importanza scientifica e pratica, sia nelle attivita civili che militali, come arl esemplo, quelli relativi all'induzione a incremento artificiale delle precipitazioni atmosforiche, al dissolvimento di strati nurolosi e della nebbia, alla climinazione o riduzione della grandine, alla eliminazione ed attenuazione di perturbazioni violente come uragani, cicloni tropicali, cece.

II. - ESPERIEXYE DI MODIFICAZIONL DELAE CONDIZIONI METEOROLOGI-

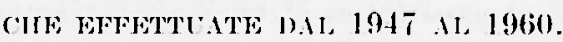

Dopo le su eitate esperienze di laboratorio di J. Tangmuir, V. J. Schacfer a guelle suceessive di B. Tonmegut (₹), furono effettuate, neggli U.S.A., dal 1947 al 1951, due serie di esperienze (ampali, ron largo uso di mezzi terrestri ed aerei, sia nelle operazioni sia nelle osservazioni dei risultati, note col nome di "Project Cirrus" a "Cloud Physies Project" Queste esperienze si possono considerare fondamentali per leulteriore evoluzione di queste ricerehe. Nellat prima serie d'esse (Project Cirrus), sovenzionata dalla organizzazione militare a affidata alla direzione di I. Langmuir e V. J. Schacefer, fu data la prova tangibile della possibilità di indure artificialmente la precipitazione, di dissolvere parzialmente o 
totalmente determinati sistemi di nubi ed, in genere, di alterare le condizioni meteorologiche naturali. I risultati pubblieati e discussi in numerosi laroli (8, 9. 10. 11, 12, 13, 14, 15) non mancearono di suscitare molte speranze e, come spesso aceade, anche molte illusioni.

La seconda serie (Cloud Physies Projeet), sostennta dalle organizzazioni meteorologiche civili degli U.S.A., mentre confermò la possibilità della alterazione dei sisteni nuvolosi, attraverso la nucleazione artificiale dell'atmosfera, denunciò la scarsa importanza economica dei risultati ottenuti e soprattutto la grande difficoltà di discernere i fenomeni naturali da quelli che poterano essere provocati artificialmente. Uno dei risultati più certi era apparso quello del dissolvimento delle nubi, in particolare condizioni di divergenza orizzontale $\left({ }^{16},{ }^{17}\right)$.

Moltre controrersie (18, 19) sollevarono le conclusioni tratte da I. Langmuir (20) circa la possibilita di effetti immediati e prolungati della nucleazione artificiale dell'atmosfera sulle condizioni meteorologiche generali e, soprattutto, sulle possibilita di modificare il clina su scala continentale. Queste allermazioni si fondavano prevalentemente su $\mathrm{m}$ esperimento condotto dallo stesso I. Langmuir $\left({ }^{21}\right)$, mediante un generatore di sostanze nucleanti artificiali (fumi di ioduro d'argento), ubicato al suolo ed operante con ma periodicita a litmo settimamale.

Dallesame dei dati pluvionetriei e delle temperature in quota, I. Langmuir aveva ritenuto di dover concludere che, alla periodicità della nucleazione artificiale dell'atmosfera effettuata, corrispondeva una analoga periodicita in vari elementi meteolologici su una rasta zona negrli U.S.A.

Contemporancamente taa il 1945 ed il 1951 furono realizzate numerose altre serie di esperienze in vari pacsi come in Australia (22, $\left.{ }^{23}\right)$, in Canada (24), in Africa orientale $\left.{ }^{25}\right)$, in Algeria (26), nello Stato di Islatele $\left({ }^{27}\right)$, in Francia $\left({ }^{28,}{ }^{29},{ }^{30}\right)$, in Italia ${ }^{31}$, 32). Il contributo di tutte queste esperienze, sebbene condotte in zone più ristrette e con minore ricehezza di mezzi di quelle degli U.S.A., diede egnalmente un utile contributo soprattutto alla conosenza delle techiche operative da usare e per la inpostazione del problema, nell'mbito delle caratteristiehe climatiche locali.

Saturalmente le controversie sui risultati telle operazioni furono tante e tali che, nel 1953, il congresso degli C.S.A. fu indotto ad istituire un ('ontitato Consultivo'(Advisory Committee on Weather Control), rol compito di studiare e valutare gli esperimenti pubblici e privati riguardanti questo campo di ricerche. La conclusione a cui gimse il suddetto "Comitaton, dopo l'esane di numerosi cicli di esperienze affidate 
a noti scienziati e ard organizzazioni sceientifiche universitalie o qualfiicate $(33,31,35,35,37,38,39,40,41,42)$ e dopo l'esame con teceniche statistiche progredite $\left({ }^{40,}{ }^{41}\right)$, dei risultati ottentuti anche dat organizzazioni commerciali, operanti sul 10\% del territorio nazionale degri U.S.A. con inprego di ingenti cappitali (si calcola rhe in quell'eporal si spendevano cirea 5.000 miliardi di lire annui), fu che, fino a quell'epoca era stato apportato un impagabile contributo allaco noscenza della fisica delle mubi e delle precipitazioni e soprattutto era stato provato che gli efietti osservati, $i$ seguito alla mucleazione artificiale della atmosfera, mppresentavano qualcosa di più delle fluttuaziomi casuali che si hamo nella precipitazione naturale. Tuttavia era ancora necessario compiere molto lavoro, con programmi a lunga scadenza, sfruttando $i$ progressi fino allora compiuti nello studio della fisica e chimica dell'atmosfera, sotto la guida di scienziati entusiasti e capaci, che fossero liberi di procedere nella direzione che il loro entusiasmo e curiositì potera consigliare, per poter giangere a concreti risultati nella soluzione del problema proposto.

Sostanzialmente, ad analoghe conchusioni giunsero anche altre organizzazioni scientifiche degli U.S.A., che si emano interessate del problema come il "Comncil of the Anerican Meteorological Society ", nel suo rapporto pubblicato nel Giugno 1957, ed il "Committee on Aceteorology of National Ararlemy of Sriences n, nel suo rapporto pubblicato nel Gennaio $1958\left({ }^{45}\right)$.

La necessita di continuare le ricerche spinse ancol"a il governo degli L.S.A. arl affidare, nel Iughio del 1958, un nuovo vasto programma di ricerche alla "National Science Fundation ". Tale programma d attualmente in atto, alla sua realizzazione sono impegnati vali istituti universitari degli U.S.A. (Arizona, Chicago, New York) a vari gruppi di ricerca qualificati. Si preverle che i risultali definitivi non si possano arere prima del 1965-66.

\section{III. - I PRIXCIPI DELLA MOIJICAZIONE ARTHFICIALE DELITE CONDIZIONI METEOROLOCICHE.}

Tenendo ora ronto:

1. - Delle teorie sulla formazione delle precipitazioni naturali, inizialmente formulate da $\Lambda$. Wegener $\left({ }^{16}\right)$, J. Bergeron $\left({ }^{47}\right)$ e Wr. Findeisen $\left({ }^{48}\right)$, per le cosidette " nubi fredrles (tutte o parzialmente a temperatilla 000 ). 
‥ - Delle critiche sui rari mecomismi proposti $(19,50,51,52)$ per spiragare l'arecrescimento degli elementi costituenti le nubi (gocce o cristalli di ghiacero), fino alle dimensioni rlegli elementi di precipitazione.

3. - Delle numerose determinazioni $\left({ }^{53}, 54,55,56\right)$ delle dimensioni, relocita e concentrazione degli alementi costituenti i vali tipi di nubi e di precipitazioni.

4. - Delle varie teorie formulate da G. C. Simpson ( $\left.{ }^{57}\right)$, I. Langmuir $\left({ }^{58}\right)$, H. G. Hougton $\left({ }^{59,}, 60\right)$, R. G. Bowen $\left({ }^{61}\right)$, F. M. Iandlam (69, 63, 6i) e B. J. Mason $\left.{ }^{65}\right)$, sulla formazione degli elementi della precipitazione per coalescenza, dagli elementi delle nubi, siano esse fredde o calcle (a temperatuma 0 or).

ว. - Delle ossererazioni e reterminazioni sui nuclei igroscopice $\left.{ }^{66}\right)$; si possono trarre le conclusioni seguenti:

a) In ma nube frerda, inizialmente, quando i eristalli di ghiacecio sono molto piccoli, il loro ingrossamento è rovuto principalmente al processo di sublimazione del vapor d'arqua.

b) Quando la grandezza dei cristalli diventa paragonabile a quella dei più piccoli elementi di precipitazione (piovigurine: 100-150 $\mu$ ), il processo di sublimazione, in relazione all'ingrossamento dei cristalli, eguaglia rome inmportanza quello di coalescenza,

c) Quando la rimensione delle particelle i ancora maggiore, prevale decisamente il processo di coalescenzal.

d) In man nube, in cui esistono poche gocere grandi, nella parte piu bassa, e il contenuto di acqua liquirla e le correnti ascendenti sono quasi stazionarie e lo spessore raggiunge qualche centinaio di metri, tali gocee, a causa dei due contemporanei effetti della condensazione del vapor d'acqua e della coalescenza, possono ingrandirsi fino a raggiungere le dimensioni delle gocee di piogrgia.

e) In molte nubi, spesso, la parte più notevole della precipitazione si può considerare doruta prevalentemente all'effetto di coalescenza delle grandi gocece o dei cristalli di ghiareceio.

Da quanto risulta dalle sudklette teorie e dalle osservazioni, si può affermare che la precipitazione atmosferica naturale si produce quando nelle nubi esiste una determinata quantità di cristalli di ghiacrio assieme a molte gocee d'acqua sopraffuse oppure esistono poche gocee d'acqua grandi fra una moltitudine di gocee più piccole. E inoltre necessario che le nubi abbiano un reterminato spessore e siano sostenute da correnti con- 
vettive di sufficiente intensità. Naturalmente da ciò no deriva, immediatamente, che se le condizioni natumali sono tali per cui o i cristalli o le gocce grandi mancano o il loro numero è insufficiente, a parità delle alt re condizioni, il processo della precipitazione si può attivare artificialmente, immettendo nelle nubi gli elementi mancanti. (io si può fare, nelle nubi fredde, introducendo sostanze irlones per la nucleazione, rhe possono essere sostanze altamente refrigelanti atte a provocare il congelamento delle goce soprafiuse e cosi produrre $\mathrm{i}$ germi di ghiacecio necessari ad attivare il meceanismo di Bergeron-Findeisen. La sostanza refrigerante più comumemente usata in questa operazione è il ghiaceio seceo $\left(\mathrm{CO}_{2}\right.$ solida ( $\left.^{*}\right)$ che può essere dispersal o, come ordinariamente si dice, "seminatal ", in nubi adatte. La "semina" si può anche realizzare mediante sostanze aventi struttura cristallina simile a quelle del ghiaceio. Ena di queste sostanze ì l'ioduro d'argento (**) (che si puo liberare nell'aria attraverso i suoi fumi, prodotti in vari modi: evaporazione diretta, bruciatura nell'aria di sue soluzioni in acetone, bruciatma di un suo miscuglio con carbonce o sostanze esplosive.

I fumi di ioduro d'argento, prodotti da appositi "generatori ", possono essere liberati in rolo o al suolo. In questo ultimo (aso, la diffusione della sostanza nucleante viene affidata all'azione delle correnti convettive dell'atmosfera.

In'altra techica, recentemente perfezionata in esperienzo condotte in Sardegna (79, 80, $\left.{ }^{11}\right)$ nel corrente anno, è quella che si serve, per la diffusione delle sostanze nucleanti ( $\mathrm{Ag}$ I e Na Cl), di particolari lazzi o cariche esplosive trasportate da palloni. Nella Fig. 1 e stata riprodotta una fotografia, eseguita in camera fredda a $-10^{\circ} \mathrm{C}$, dei rristalli di ghiaceio formati sui nuclei dei fumi di $\mathrm{AgT}$ delle miscele esplosive impiegate. Sono stati calcolati per ogni grammo di $\mathrm{AgT}, 1,6 \cdot 10^{1-}$ nuclei dopo 6 minuti.

Sel aso delle nubi calde, la nucleazione artificiale si può realizzale immettendo, alla base delle nubi da trattare, grandi goce d'arqua, mediante spruzzatori trasportati da aeroplani, oppure, mediante dispersione di sostanze altamente igroscopiche atte a produrre nuclei di condensa-

$\left({ }^{*}\right)$ Secondo I. Langmuir una pallina di ghiarecio seceo di 5 mm di diametro puo produrre $10^{16}$ eristalli di shiacio d'acqua, mentre Weichmann afferma ehe an grammo di ghiaceio seceo può produrre $10^{9}$ eristalli di ghiaceio d'acqua. lamento.

$\left.{ }^{* *}\right)$ Un grammo di $\operatorname{Ag} \mathrm{I}$ puó produre da $10^{12}$ a $10^{15}$ nuelei di conge- 


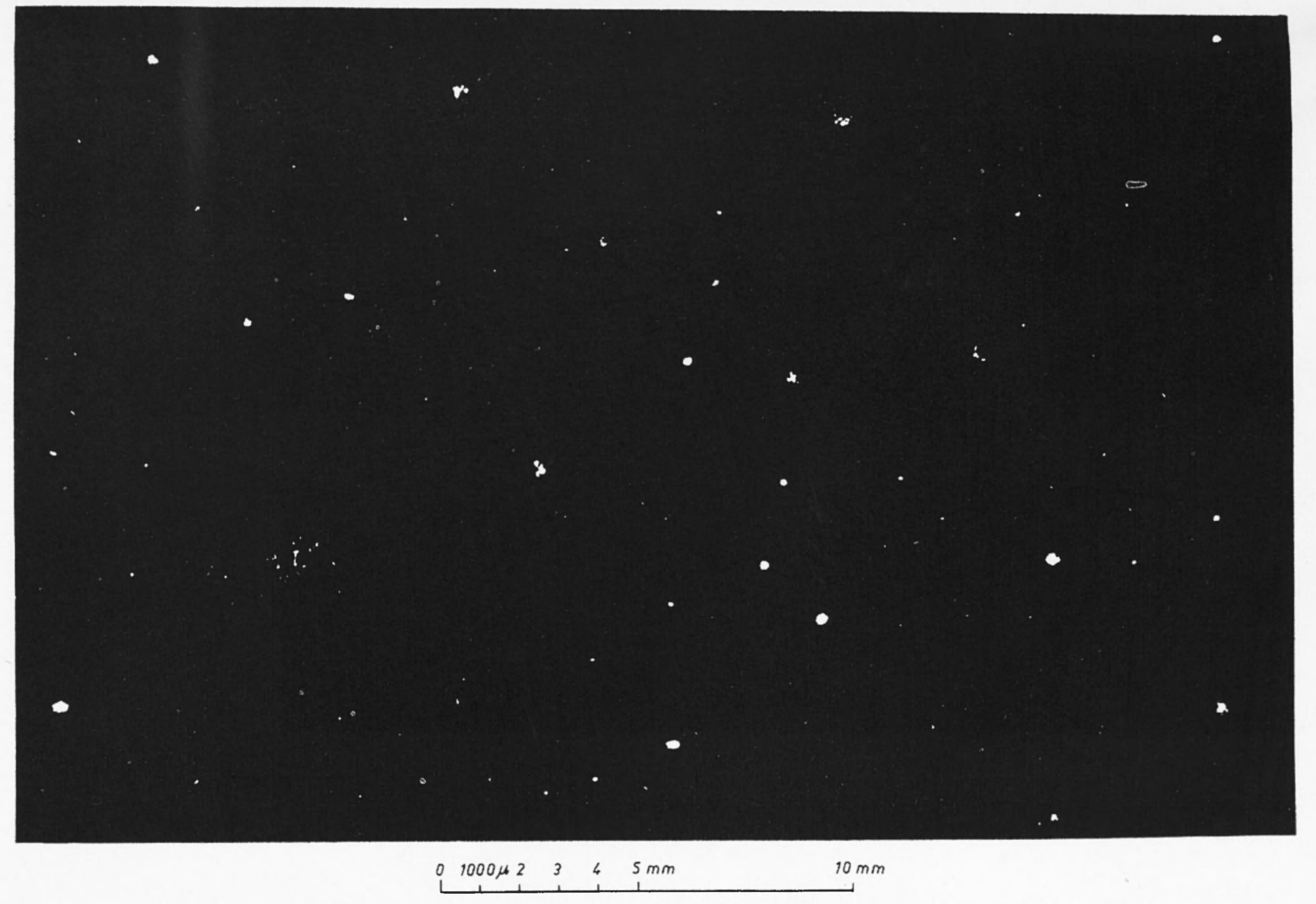

Fig. I - Cristalli di ghiaceio ottenuti in camera fredda con nuclei di ioduro d'argento. Temperatura -10 of. 
zione griganti. La dispersione, in questo aso, si può fare da aeroplani, da palloni o razzi ed anche dal suolo, riducendo la sostanza nucleante in particelle submicroscopiche ed affidandone la diffusione alle correnti convettive dell'atmosferi.

La teconica delle goce d'acqua e dei nuclei igroscopici fu tentata ancom prima che si conoscesse la teoria della precipitazione per coalescenza. Aleuni esperimenti furono tentati nell'Ohio in U.S.A. $\left({ }^{67}\right)$ e nell'Africa del Sud $\left({ }^{68}\right)$ già nel 1948. Anche le esperienze fatte nel 1948 nelle Hawai ("') ('on ghiaceio seceo, in nubi calde, furono interpretate da I. Langmuir, sulla base di un processo di coalescenza sulle gocee d'acqua giganti, formatesi per condensazione del vapor d'acqua sui granelli di ghiaceio secero.

Solo nel 1950, dopo la trattazione teoriea sulla coalescenza, elaborata da F. H. Ludlam ( $\left.{ }^{6}\right)$, E. G. Bowen $\left(^{(6)}\right.$ ) realizzò in Australia le prime esperienze sistematiche immettendo, alla base delle nubi, grandi gocee d'accua. Successivamente lo stesso Bowen (50) nel 1951-52, potè in un altro ciclo di esperienze constatare un buon accordo con la teoria.

Un sistema più economico è quello di introdurre alla base delle nubi, invece di gocee d'acqua, nuclei di sostanze igroscopiche sui quali si possono formare le goece necessanie. Cosi su nuclei di sale marino, della dimensione di $10 \mu$ di diametro, si possono, in breve tempo ed in aldatte condizioni di umidità, formare goce di dimensioni doppie atte ad attivare il processo di coalescenza. D'altra parte si calcola che $150 \mathrm{grammi}$ di sale marino possono equivalere a 5 litri di acqua spruzzata a gocese di $50 \mu$ di diametro.

Nella Fig. 2 è riportato l'ingrandimento di tre microfotografie della formazione di gocee d'acqua giganti su cristalli di sale marino, impiegato come sostanza nucleante nelle esperienze condotte in Sardegna $\left({ }^{79},{ }^{80},{ }^{81}\right)$ nel corrente anno, con la tecnica dei razzi. Si può notare che su un agglomerato di cristalli della dimensione di circa $100 \mu$ di diametro si è formata in 12 secondi una goceia di $225 \mu$ di diametro ed in 20 seconti di $250 \mu$, con una umidità relativa del $96 \%$ \%

Particolari tecniche di dispersione di nuclei igroscopici, nelle nubi, furono realizzate, mediante soluzioni saline atomizzate in Francia, da H. Dessen ( $\left.{ }^{11}\right)$, nel 1950; mediante miscele esplosive, trasportate da palloni, da D. A. Davies ed altri $\left.{ }^{(2)}\right)$ nel 1951, in Afriea Orientale, e, mediante dispersione dal suolo sfruttando le correnti convettive, da J. M. Fornier d'Albe nel Pakistan nel 1954 e più recentemente nel Messico (73, $\left.{ }^{71}\right)$.

La nucleazione artificiale dell'atmosfera, mentre con determinate condizioni dinamiche e termodinamiche delle nubi, può indurre la pres- 


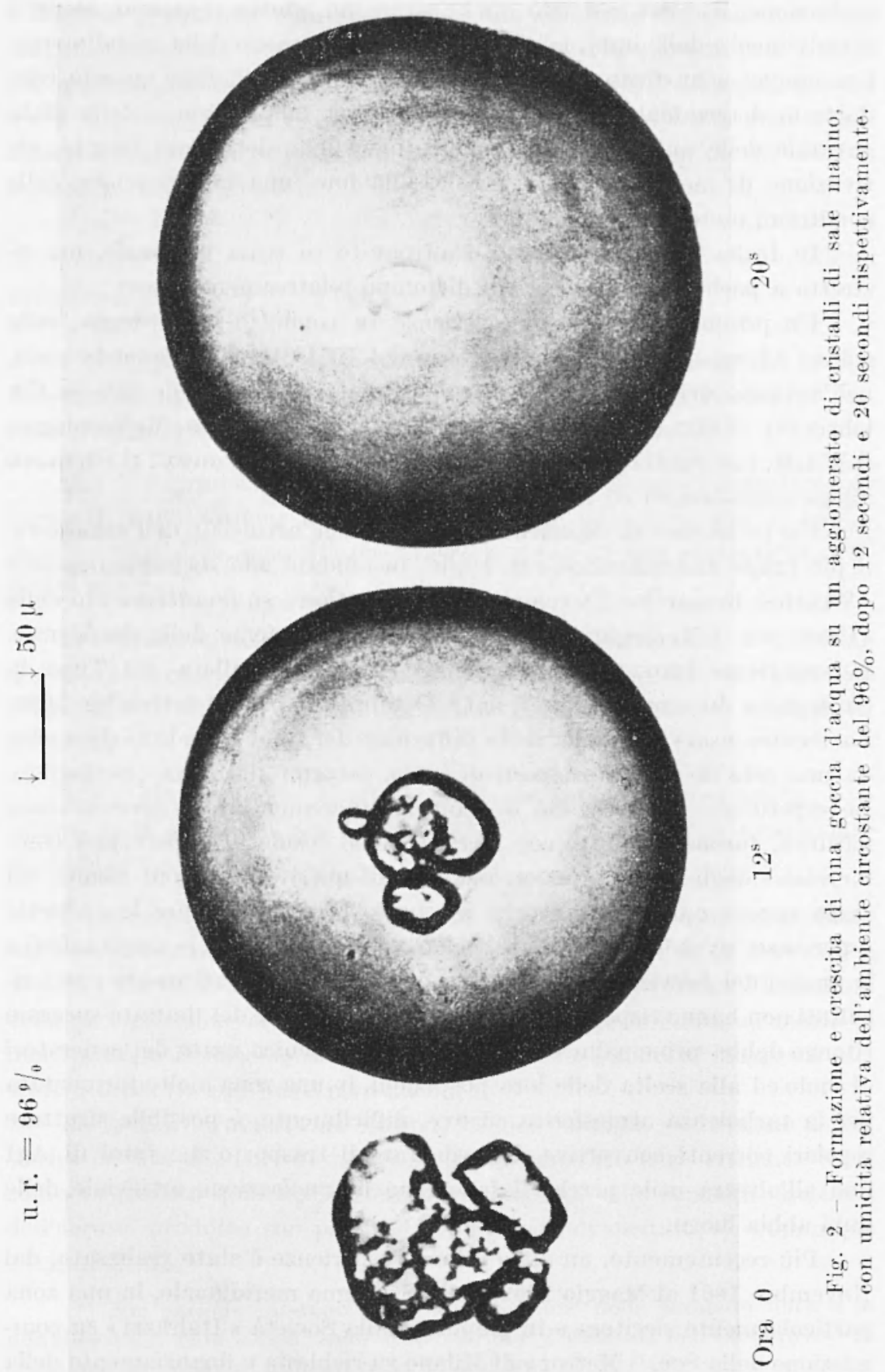


riphitazione, in altri casi può anche avere un effetto contrario, come il dissolvimento delle nubi, la prevenzione del fenomeno della grandine ece. Comunque, d un fatto certo che la nucleazione artificiale, quando condotta in determinate condizioni, provoca una modificizione dello stato naturale delle nubi, con conseguente liberazione del calore latente, attivazione di moti convettivi, e rioè, alla fine, ma modificazione delle rondizioni metrorologiche.

In Italia il problema è stato affrontato su scala nazionale, ma ristretto a poche località e periodi di tempo relativamente brevi.

Un primo breve ciclo di esperienze fu condotto in Sardegna, sulle colline ad est di Cagliari, nell'inverno del $1951{ }^{31}$ ) ed un secondo ciclo, nell'autunno dell'anno seguente, sull'altipiano della Grande Sila in Calabria $\left(^{32}\right)$. Entrambi i cicli furono diretti dal Servizio Meteorologico dell A.M. con risultati che, dato il modesto impiego di mezzi, si ritennero allora soddisfarenti ed incoraggianti.

Un terzo ciclo di esperienze di nucleazione artificiale dell'atmosfera, il piì lungo finom condotto in Italia, fu afficlato alla società americana "Weather Researches Development Corporation" su finanziamento della "Cassa per il Mezzogiomo" e la "Regione Autonoma della Sardegna ". Le esperienze furono effettuate lungo la media vallata del Tirso in Sardegna e durarono tre anni, dal 1 Ottoble 1957 al 30 Settembre 1960. Ia techiea usata fu quella della diffusione dei fumi di ioduro d'argento da una rete di generatori posti al suolo, attorno alla zona "bersaglio ". Le esperienze, che avevano lo scopo di incrementare le precipitazioni naturali, furono condotte eon i criteri delle cosidette "operazioni commerciali " degli U.S.A. Nessun risultato di queste operazioni risulta sia stato ancora pubblimato. Poiché ho avuto modo di seguire le suddette esperienze, avendo la suddetta Società potuto utilizzare le osservazioni e le analisi del Servizio Meteorologico dell'A.M., posso affermare che i risultati non hanno risposto alle aspettative. La causa del limitato suceesso ritengo debba principalmente attribuirsi alla teenica usata dei generatori al suolo ed alla scelta delle loro postazioni, in una zona molto tormentata per la turbolenza atmosferica ed ove, difficilmente, è possibile sfruttare regolari correnti convettive da assicurare il trasporto dei fumi di $\mathrm{AgI}$ fino all'altezza utile perchè il fenomeno di nucleazione artificiale delle nubi abbia luogo.

Più recentemente, un altro ciclo di esperienze è stato realizzato, dal Norembre 1961 al Maggio 1962, nella Sardegna meridionale, in una zona particolarmente siccitosa e in pianum, dalla Società "Italrazzi " su commissione della Soc. "Meteor" di Milano su richiesta e finanziamento della 
"Regione Autonoma della Sardegna ". La direzione scientitica è stata afficlata al Servizio Meteorologico dell' A.M.. Della tecrnica usata si c̀ fatto gia qualche fugace remo. I risultati sono ancora allo studio, ma fin d'ora si possono ritenere incoraggianti, nonostante si trovi grande difficoltà a trare conclusioni definitive, in senso statistico, da un ciclo di esperienze cosi breve (*).

\section{Coxchintone.}

I principali aspetti del problema, che ancora non hamno aruto una soddisfacente soluzione e che meritano ulteriore aceurata ricerea, si possono riassumere breremente nei seguenti punti:

1. - Determinazione della quantità esatta di nuclei di congelamento esistenti nell'atmosfera in diverse condizioni meteorologiche e, conseguentemente, della quantità occorrente per una efficace nucleazione artificiale.

2. - Determinazione della diffusione e concentrazione, a varie distanze ed altezze, in differenti condizioni meteorologiches, dei nuclei di congelamento efficaci, nei fumi di ioduro di argento liberati al suolo ed in quota.

3. - Valutazione dei risultati, mediante determinazioni fisiche, particolamente quanclo le più raffinate teconiche statistiche non sono idonece all una valutazione esatta.

Le ricerche, per quanto riguardano il primo punto, sembra debbano mettersi in relazione, anche alla attività delle meteoriti. E. G. Bowen (i⿱5) nel 1953 aveva notato che, fra le date in cui si erano verificati i massimi della precipitazione atmosferica osservata su tutta la Terra, su medie di molti anni, e Ie date spostate di 30 giomini delle cosidette piogge di meteoriti, esisteva una forte correlazione.

La ipotesi formulata da Bowen, su una possibile mucleazione atmosferica dovuta alle polveri meteoritiche, ha sollevato varie critiche (76, 7 ), come ha trovato qualche possibilità di conferma sulle capacità nucleanti dellarerosol prodotto con particelle volatizzate di metalli (78). Commoue

(*) Sel periodo intereorso fra la esposizione della presente nota e la pubblicazione della stessa, sono stati resi noti $\mathrm{i}$ risultati delle esperienze in questione in tre lavori indieati nella bihliografial $\left.{ }^{79}, 80,81\right)$. 
¿ molto interessante stabilire con certezzal se le nubi siano o no seminate dal pulviscolo meteoritieo ed in qual misura, pere potere, eventualmente, intererenire artificialmente nei periogi in cui mancal ma tale semina naturale. Saturalmente, il problema non è semplice, in cquanto è necesssalro determinare non solo la quantita ma anche le dimensioni, forma e natura chimica e tempo di sedimento di tale materiale extraterestre. E questo tutto un nuoro campo di studio in chi la tecenica delle osservazioni spaziali potrà dire una parola definitiva.

Ci possiamo om rhiedere quali siano lo attuali possibilita della nureazione artificiale dell'atmosfera?

Esaminando la possibilita di realizzare un incremento della precipitazione su scala mondiale si può subito affermare che ma simile ipotesi debba essere attualmente scartata. Infatti, la slat realizzazione significherebbe mala accelerazione del riclo idrologico atmosferico, costituito dalle tre fomelamentali fasi della evaporazione, condensazione e precipitazione. Si dovrebbe cioè provorare mal accelerazione nel moto della macrehina atmosferica, che essendo, come è noto, alimentata da continua fornitura di energia esterna (principalmente la radiazione solare), sarebbe di reosto immenso.

Esiste pereio, almeno per ora, solo la possibilità di operazioni di nucleazione artificiale dellarmesfera, tali da consentire una ridistribuzione delle precipitazioni a scala limitata ad es. su parte dei continenti. Tale ridistribuzione si oftermebbe provocando precipitazioni premature o aumentando o riducendo la precipitazione naturale sui continenti, rispetto a quella che carle sui mari.

Orviamente, piani di esperienze di tale entità comportano, necesssariamente, una vasta organizzazione internazionale (s soppattutto la conoscenza della distribuzione della nurolosita e dei rentri di perturbazione in una scala emisferica, che oggi si potrebbe oftenere attraverso lo sfruttamento delle osservazioni compiute da satelliti artificiali.

Analogamente, si possono concepire operazioni di nucleazione artificiale dell'atmosfera tendenti ad incrementare le precipitazioni su regnioni aride o semi aride, a spese di altre regioni più picrose. Questo problema, più limitato del precedente, riducibile a scala mazionale o regionale, pressenta, anche esso, molte difficoltà a non può presseindere dat man profonda conoscenza della climatologia dinamica della zona da trattare e di un suo intorno sufficientemente vasto.

Risultati molto promettenti delle teconiche di nucleazione artificiale dell'atmosfera, finoma arloperate, per riconoscemento quasi unanime, si sono avuti dalle cosidette formazioni nurolose orografiche e semiono- 
grafiches, rioè legate alla presenza di aatene di montagne e di colline sufficientemente alte, mentre nelle zone di pianuma i risultati oftenuti non somo statisticamente apprezzabili. Ciò potrebbe far pensale alla possibilità di operare su regioni di montagna o di collina, al fine di creare riserve idriche in bacini di raceolta, oceorrenti poi per la imigazione delle zone piane. Son vi è dubbio, alla luee dell'attuale conoscenza, che esperinenti del genere siano promettenti di fecondi risultati, sia nel (ampo della ricerea puramente scientifica che delle pratiche applicazioni.

Rivolgendo uno sgualdo al passato anche da quanto mi risulta da personale esperienza, non posso che contormare quanto ebbi a dire undie anni or sono, quando cominciai ad interessarmi di queste riecredere, dioe d'essere convinto della possibilità di una favorevole soluzione di questo problema, che tanta utilità puó apportare alla umanità. Xon credo, oggri, d'essere lontano dalla realtà affermando dhe il prossimo decemio vedrà enormemente ampliata la comosecenza nel campo della disica delle nubi e. delle precipitazioni con la definitiva determinazione delle possibiliti umane in questo difficile campo del governo del tempos.

\section{BIBILIO)(:RATIA}

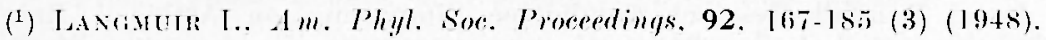

$\left({ }^{2}\right)$ Schaterer V, J.. Scrience, 104, 457.459, Nov. 19+6.

(3) Esps .I. P., Philesophy of storm. Boston l $8+1$.

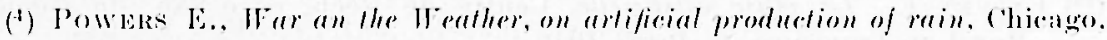
S. C (iriggs: and (O). (ISTI).

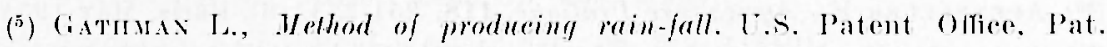
11" +62795. 10 Nox. 1891: lierin produced al will. Chicago 1891.

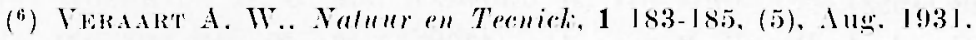

(₹) Voxiciut B., Jour. of Appl. Physies, 18, 593-595, (7), Jul, 19+7.

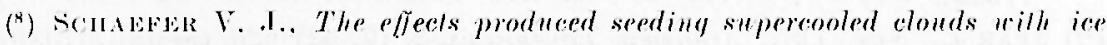
and silver iodide. Procesest. Cent. Roy. Met. Sor. (1950).

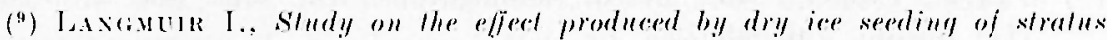
clouds. Cien. Elece. Res. Lahb., Schenectady, Occasional Report on Project Cirrus, 10, Feb. 1949.

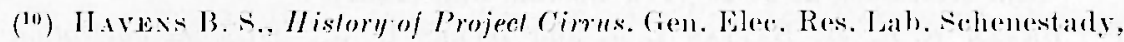
x. Y. Report No RL, 756, Jul. 1952.

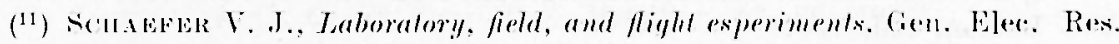
Lab. Contract D.A. 36-039. Sce 15+5. Project DA 3-90-07-022 (Project ('irrus) - Final Report Pt. I Mareh 1953.

(12) Laximule I., Science, 112, 35 (1950). 
(13) Lavinuen I., Progress in olouds modificalions by Projert Cirms. Cien. Eler. Res. Lahh. Project (irrus - Final Report, 259, (1951).

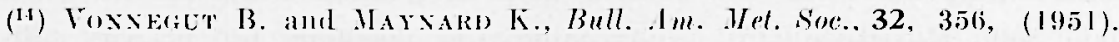

(15) Voxigeter B., Scientifie Imariean, 186, 17-22 (1) Jan. 1952.

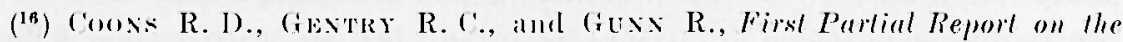
artificial produstion of mecipilation: stralisform slonds. Ohio, 1948. U. S. Weather Bureau-Research Paper. 30. Aug. 1948.

(17) Cooss R. D., Josws E. I. and (ivxx R.. Second, Thim, Fourth-Partial Report on arfificial production of presipilation. Cumuliform Clouts, Ohio 1948; Orografie stratiform Clouds-California, 1949) Cumulus Clouds, Gulf States, 1949, U.S. Weather Bureau Researeh Paper, 31, (1949), 32, (1949): 33, (1949).

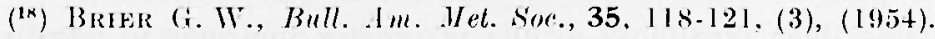

(19) Brter (4. W., Bull. Am. Wet. Sore, 36, 265-277, (6), (1955).

(20) Laxigulr I., Science, Wash. D.C., 112, (2898), 34-51, (1950).

(21) L.Axciuvir I., Bull. Am. Hel. Soce, 31, 386i-387, (10), (1950).

(22) Bowkx E. (i., IVealler, 7, 204, (1952).

(23) Bowkx E. (i., Bull. Am. Mel. Soc., 33, 24+-246, (6), (1952).

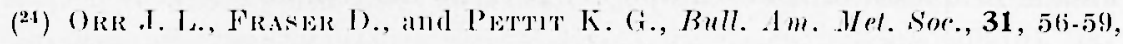
(11), (1950).

(25) Davas D. A.. Yature. Lomdon, 174, (4+23), 256-25s, (1954).

${ }^{(26)}$ Serpolay R., Bull. Obs. Puv de Dome, Ser 2, 1. 27-31, Jan. 1953.

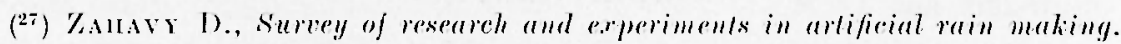
Israel Raiufall Researeh Committer simposium on Artificial Rain, Halisiral, 49-55, Aug. 1951.

(28) Erkaud R., Ae. Sci. Paris, Comp. Ren. 226. 829-831, Mar. 1948.

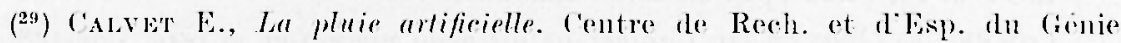
Rural et Traveaux. 9, Paris 1949.

(30) Apwoldak F., Agricollure Pralique, 118, 251-253, (5), Paris, May 1954.

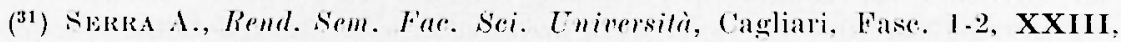
$27.29(1953)$.

(32) Eva (a. ed al. Riv di lleteor. Aer., 13, 3-15 (1). Roma, Gemn.-Marzo (1)53).

(93) SpAk J., New Tork Academy Trans. Ser. 2. 17, 621-626. (8) Jun. (1955).

(3) Spar J., Project Scudl. Meteor. Monographs. Am. Met. Soe. 2, 5-23. (II) Boston, Jul. (1957).

(35) Hald W. F., The Wealler Burean ION Project. Meteor. Monographs. Am. Met. Soc., 2, 2t-26 (11), Boston, Jul. (1957).

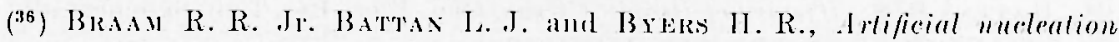
of cumulus clouds. Meteor. Monographs-An. Met. Noc.. 2 (11) $47-85$, Boston. Jul. 1957.

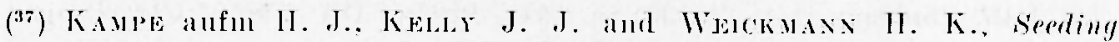
erperiments in subcooled stralus clouds. Meteor. Monograplis. Ant. llet. soc. 2. 8(j-111 (11), Bustoul, Jul. (1957). 
(3n) Pateressex \$., I Reporls on experimenls wilh arlificial cloud nuclealion. Meteor. Monographis.An. Met. Soe. 2, 4. (11). Boston. Jul. 1957.

(39) ORwiles H. T., Summary of Commillee's Findings and Recommandalions. Final Report of the Advisory on Weather Control. I, I0. Washingtoll 26 I).C. Dec. 31, (1957).

(40) Boucisis R., Operalion overseed. Final Report of the Advisory Commitese on Weather ('ontrol, II. 127-136, Washington D).C., 31 Dic. (1957).

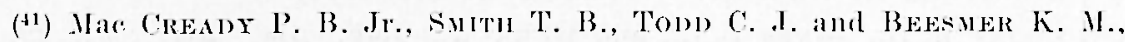
Nuclei, cumulus, and seadibility sludies. Final Report of the dovisory committee on Weather Control, II, 137-200, Washington D.C.. 31 l)ic. (1957).

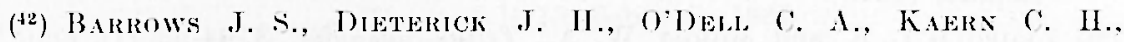

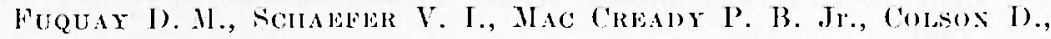
Wress H. J.. Project skyfire. Final Report of the Adrinory Commitese on Weather Control, II, 105-125. Washington D.C., 31 Dic. 1957.

(43) 'THon H. C.S.. A slalistical melhod of evolution angmentalion of precipitation by cloud seeding. Final Report. of the Adrisory Committee on Weather Control II, Technical Report N. 1, 5-25. Washington I).C.. 31 Dic. 1957.

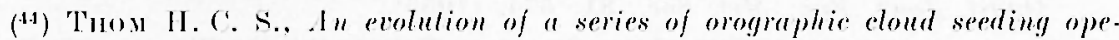
ralions. Final Report of the Advisory Committee on Weather control II. Techuical. Report. No 2, 25-50, Washington D.C., 31 Dic. 1957.

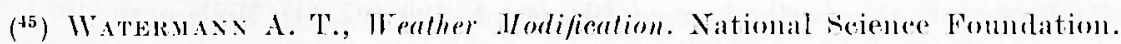
First Annual Report. 5, Washington D.C., .Imı. I960.

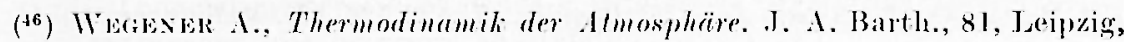
(I8II).

(17) BERGERox J., On the physics of elouds and precipilation. Proce. 5th Assembly l'.(4.G.L., 156. Lishon, 2 (1935).

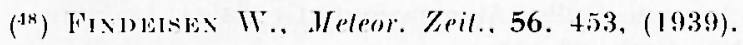

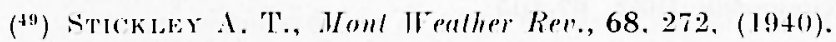

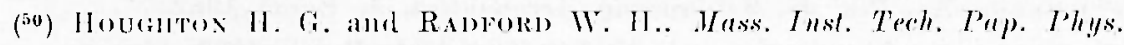
Ocean. Met.. 6. (4), (1938).

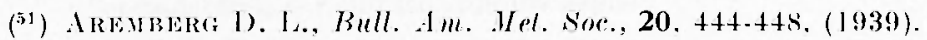

(52) FINDELAX W., Meleor. Zeil., 56, 365. (1939).

(53) Juxie ('., Ber. Dhseh. Wellerdiensl. U. S. Zone, 35, 261, (1952).

(5.1) Wвгекахх I. К., Jour. of Mel., 10. 204, (1953).

(55) 1)tis M.. Meleor. Rumdschau. 1. 261. (19+8).

$\left.{ }^{56}\right)$ Kanple aufn H. .J. Das Weller und scime Vraghen. Darmstadt. Dietrich Siteiuliojeff, 1951 .

(57) Stupsox (․ ('., Quart. Jour. Roy. Met. Soc., 67, 99) (1941).

(58) Jaxisuou I., Jour. of Mel.. 5, 175. (1948).

$\left.{ }^{59}\right)$ Housitos H. G.. On the physics of clouds and precipilation. (onupendium of Meteorology Am. Met. Sox. 176. Boston. (1951). 
(60) HovgitTox II. (i., Jour. of . Vet., 7, 3633-3699. (1950).

(61) Bowex R. (1., Austr. Jour. Nei. Res., 3. 193, (1950).

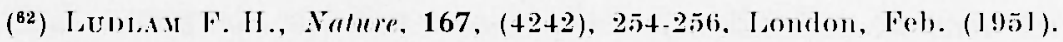

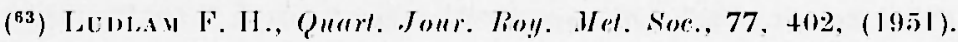

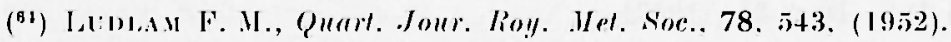

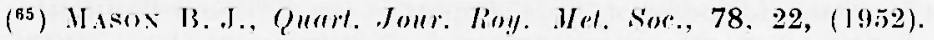

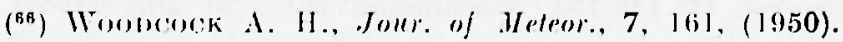

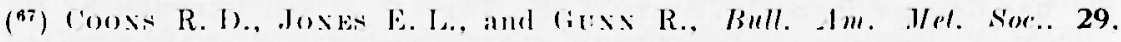
$206(19+8)$.

("6) Anonimo, Arlificial simulation of precipilation. S. Afriein C.S.I.R. Report, (19+8).

(99) LEOPOLI) L. B. and MorDY W. A., Tellus-shokholm.. 3, 4+-52, (1), Febr. I9:1.

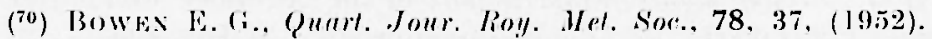

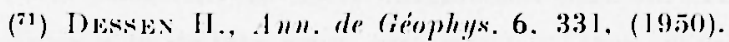

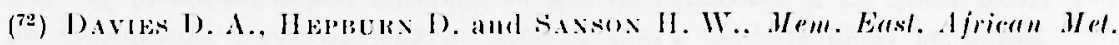
Hep. 2, 9, (1951).

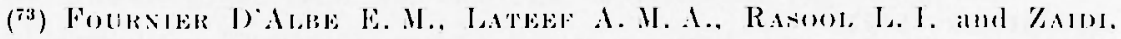
Quarl. Jour. Roy. Vel. S'ser., 81, 57t, (1950).

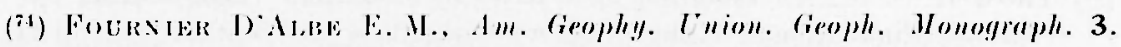
$106-110$, (1959).

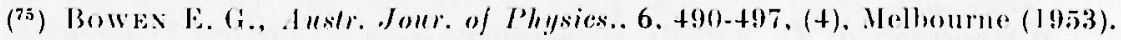

(76) MARTYo I). F., dustr. Jour. of Physies., 7, 358-364. (2), Melhourne (1954).

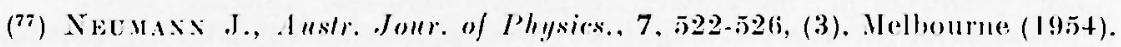

(78) Sskpotar R., Bull. Ols. Puy. de Dome. Ser. 2, 3, 87, Jul..Sept. (1950).

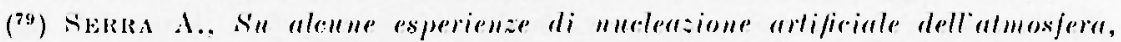

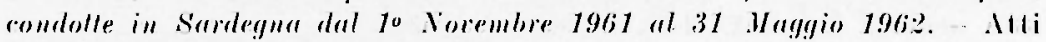

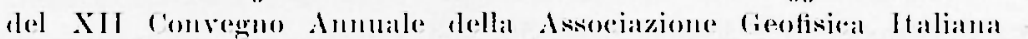
Roma 23-2t Novembre 1962, 67-105.

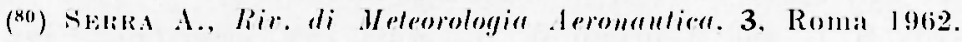

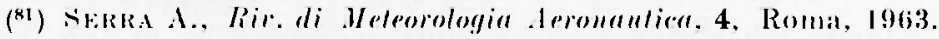

